\title{
The role of chemokines in the pathogenesis of neurotropic flaviviruses
}

\author{
Susana V. Bardina · Jean K. Lim
}

(C) Springer Science+Business Media, LLC 2012

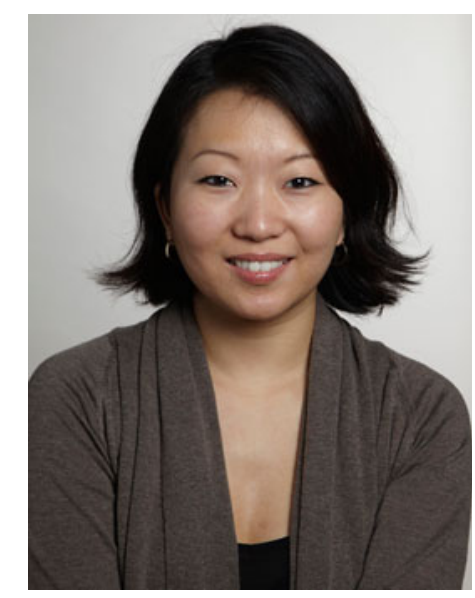

Jean K. Lim

\begin{abstract}
Neurotropic flaviviruses are important emerging and reemerging arthropod-borne pathogens that cause significant morbidity and mortality in humans and other vertebrates worldwide. Upon entry and infection of the CNS, these viruses can induce a rapid inflammatory response characterized by the infiltration of leukocytes into the brain parenchyma. Chemokines and their receptors are involved in coordinating complex leukocyte trafficking patterns that regulate viral pathogenesis in vivo. In this review, we will summarize the current literature on the role of chemokines in regulating the pathogenesis of West Nile, Japanese encephalitis, and tick-borne encephalitis virus infections in mouse models and humans. Understanding how viral infections trigger chemokines, the key cellular events that occur during the infection process, as well as the immunopathogenic role of these cells, are critical areas of research that may ultimately guide a much needed effort toward developing specific immunomodulators and/or antiviral therapeutics.
\end{abstract}

Keywords Chemoattractant $\cdot$ Leukocyte trafficking $\cdot$ Neuroinvasion $\cdot$ Immunopathogenesis $\cdot$ Viral encephalitis

\section{Introduction}

The Flavivirus genus in the Flaviviridae family consists of over 70 small $(40-65 \mathrm{~nm})$, positive sense single-stranded RNA enveloped viruses that are approximately $11 \mathrm{~kb}$ in length. This genus includes several globally important human pathogens, many of which are neuroinvasive and cause significant morbidity and mortality in their infected hosts. The neuropathogenic mechanisms employed by these viruses are a critical area of research due to the lack of specific antiviral treatments. Due to changes in climate and other anthropogenic factors that perturb the dynamics among vectors, amplifying hosts and humans, these viruses may have an enhanced potential to spread and cause human outbreaks. The most recent and dramatic example of this comes from the well-documented introduction and spread of West Nile virus (WNV) in North America, an epidemic

S. V. Bardina · J. K. Lim $(\bowtie)$

Department of Microbiology, Mount Sinai School of Medicine,

1 Gustave L. Levy Place, Box 1124, New York, NY 10029, USA

e-mail: jean.lim@mssm.edu that started in 1999 and is now the dominant vector-borne viral pathogen and the leading cause of arboviral encephalitis in the US [1, 2]. Japanese encephalitis virus (JEV), the most frequent cause of mosquito-borne encephalitis worldwide, has been progressively expanding its geographic distribution to the Western Pacific (reviewed in [3]). Tick-borne encephalitis virus (TBEV), which has historically been endemic to Russia and Central Europe, has emerged in several Western European countries in recent decades [4]. It is clear that neuropathogenic viruses pose an unpredictable global threat, and understanding their pathogenesis is an important first step toward the development of effective therapeutic strategies.

\section{Pathogenesis of neurotropic flaviviruses}

Flaviviruses that cause neurological disease in humans are transmitted either by ticks or mosquitoes. All of the mosquito-borne encephalitic flaviviruses are classified antigenically into the JE serocomplex, while the tick-borne flaviviruses belong to the tick-borne encephalitis serocomplex. Natural infection typically starts with the bite of 
an infected arthropod, although other transmission mechanisms have also been documented [5-7]. The specific mechanisms of pathogenicity for $\mathrm{WNV}, \mathrm{JEV}$, and TBEV in humans are largely unknown, but are likely to have both overlapping and distinct features. Excellent in vivo models have provided insight into the various components of the innate and adaptive immune response critical for virus clearance. In these models, virus replication occurs locally in the skin and draining lymph nodes, which results in viremia and dissemination of the virus to extraneural tissues including the spleen. In some cases, the virus is capable of breaching the blood-brain barrier (BBB), where it replicates primarily in neurons. The precise mode underlying viral entry into the CNS during natural infection remains unclear but is likely to be influenced by multiple factors related to both the virus and the host. The spectrum of clinical outcomes following infection is broad, with most infected individuals remaining asymptomatic. The clinical syndromes that arise are also heterogeneous; mild symptoms typically include fever, malaise, and headache and are often self-limiting, whereas severe neuroinvasive disease includes meningitis, encephalitis, and myelitis, with mortality rates among encephalitic patients as high as $50 \%$ (reviewed in [3]). Surviving individuals often suffer from long-term neurological sequelae. Understanding not only the host factors that dictate susceptibility within a population infected with a particular virus but also elucidating common pathogenic strategies utilized between these flaviviruses is a vital area of research.

\section{Chemokines and leukocyte trafficking}

Encephalitis is the most serious disease manifestation caused by neurotropic flaviviruses and is characterized by the presence of leukocytes into the brain parenchyma, which is observed in both infected human and animal models. It is generally accepted that chemokines, a superfamily of chemoattractant cytokines, are involved in regulating complex leukocyte trafficking patterns during infection that are essential components of cell-mediated immunity and antiviral host defense. Chemokines exert their effect by binding and signaling through their cognate receptors, which are found differentially expressed on all leukocytes. Despite great redundancy within the system, chemokines are capable of mediating complex biological functions with exquisite specificity in response to pathogens. This is owing to not only the dynamic chemokine receptor expression patterns on specific cell subpopulations during an inflammatory response, but also the timing and composition of the chemokines induced. Furthermore, binding of chemokines to glycosaminoglycans, posttranslational processing events, and the tissue milieu in which the infection occurs all contribute to the unique in vivo host inflammatory response. In the following sections, we will review what is currently known regarding leukocyte trafficking and chemokine induction during WNV, JEV, and TBEV infections and their contribution to the unique pathogenesis of these viruses.

\section{West Nile virus}

WNV is maintained naturally between mosquitoes and birds, although other vertebrates can also become infected, including humans and horses. WNV was initially isolated from the blood of a febrile woman in the West Nile province of Uganda in 1937 and since has caused sporadic outbreaks of a mild febrile illness with low incidence of neurological complications in regions of Africa, the Middle East, Asia, and Australia [8, 9]. However, in the mid1990s, the epidemiology and ecology changed, with frequent outbreaks occurring in new geographic areas characterized by high rates of severe neurological disease and death. In 1999, WNV was identified as the causative agent of seven human fatalities as well as a large number of avian deaths in New York City and the surrounding areas [10, 11]. From there, the virus spread westward across the US and into Canada, and southward into Mexico, the Caribbean, and Central/South America, decimating bird populations and causing 31,257 cases and 1,254 (4\%) associated deaths $[12,13]$. WNV has been detected in over 300 bird and 60 mosquito species in North America and appears to be firmly implanted in the New World [10]. The success of WNV can be attributed to several factors, such as the presence of numerous competent Culex species for transmission, and the naive and highly susceptible bird populations of North America. Now endemic, WNV is the leading cause of arboviral encephalitis in the US, and the need for antivirals and an effective vaccine for humans is paramount [11]. In humans, most WNV infections are asymptomatic. Symptoms arise 2-14 days postinfection in $\sim 20 \%$ of infected individuals and can range from a mild flu-like illness, known as West Nile fever (WNF), to more serious neurological complications, including meningitis, encephalitis, and acute-flaccid paralysis. Approximately, 1 in 150 infected individuals will develop neuroinvasive disease. Mortality is $\sim 10 \%$ among individuals who develop neuroinvasive disease, and postneurological conditions are common ( $>50 \%$ ) (reviewed in [14]). Although immunocompetent individuals of all ages can develop neurological complications, increased age and immunosuppression are strong risk factors for severe outcome with WNV infection. This can also be recapitulated in mice infected with WNV, which suggests that an intact and robust immune response is required at the early stages of 
infection prior to CNS entry as well as late stages after virus has entered the CNS compartment $[11,15]$.

In mice, intradermal or subcutaneous WNV inoculation results in limited viral replication in the skin and draining lymph node [16-18]. Virus then reaches the blood stream and can access various peripheral organs, such as the spleen, and in some cases cross the BBB [18-20]. Once in the CNS, WNV primarily infects neurons in the cerebral cortex, brainstem, basal ganglia, and spinal cord [21]. This pattern of virus replication in vivo is hallmarked by a sequential pattern of chemokine production followed by distinct cell trafficking events as shown in Fig. 1. Although in vitro studies have demonstrated infection in both astrocytes and neurons, in vivo studies in mice and human autopsy tissues show infection exclusively in neurons [22, 23]. As shown in Table 1, the most reproducibly induced chemokines in the CNS in response to WNV infection in vivo are the CCR5 ligands (CCL3, CCL4, and CCL5), CXCR3 ligands (CXCL9, CXCL10), and the CCR2 ligands (CCL2, CCL7, and CCL12) [24-28]. In addition, changes in CCR7 ligands (CCL19 and CCL21), CXCL12, and CXCL14 have also been observed [24, 25, 29]. Microglia are poorly permissive to $\mathrm{WNV}$ in vitro but can produce robust amounts of chemokines in response to WNV with a similar profile of induction, including CXCL10, CCL2, and CCL5 [30]. In humans, histological examination of CNS tissues from autopsy samples shows primarily $\mathrm{T}$ cells in the brain parenchyma and a mixture of neutrophils and $\mathrm{T}$ cells in the cerebral spinal fluid (CSF) [31-35]. In mice, WNVinfected CNS is comprised of cellular infiltrates composed of CD4+ and CD8+ T cells, NK cells, neutrophils, and infiltrating monocytes $[25,26,36]$. To start addressing the

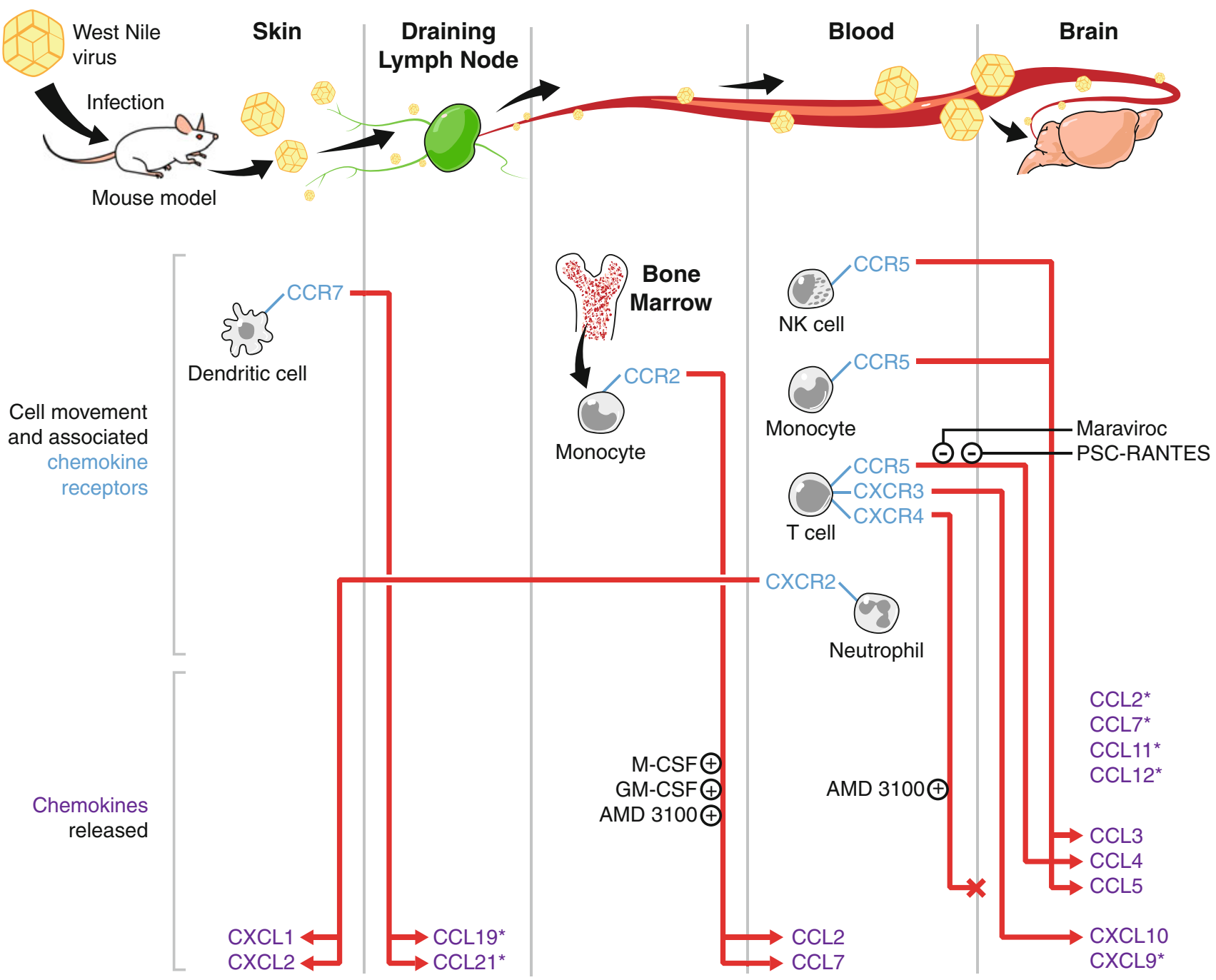

Fig. 1 The role of chemokines in WNV pathogenesis in vivo. In the experimental mouse model of WNV, chemokines are induced within specific organs during infection as indicated in purple. Also shown are chemokine receptors in blue and subsequent immune cell

recruitment denoted by red arrows. Potential therapeutic targets that may promote or inhibit specific trafficking events in vivo are shown. Asterisks denote chemokines not fully characterized in this model 
Table 1 In vivo chemokine induction during WNV infection

\begin{tabular}{|c|c|c|c|c|}
\hline Compartment & Species & Cells & Chemokines & Reference \\
\hline \multicolumn{5}{|l|}{ WNV } \\
\hline \multirow[t]{2}{*}{ Blood } & Human & - & CCL2, CXCL9, CXCL10 & [28] \\
\hline & Mouse & Monocytes & CCL2, CCL7, CCL12 & [27] \\
\hline CSF & Human & Neutrophils and T cells & - & {$[32-35]$} \\
\hline \multirow[t]{2}{*}{ Brain } & Human & $\begin{array}{l}\text { Predominantly CD8+ T, fewer } \\
\text { CD4+ T and B cells }\end{array}$ & - & {$[31,32]$} \\
\hline & Mouse & $\begin{array}{l}\text { CD8+ and CD4+ T cells, } \\
\text { monocytes, neutrophils, NK cells }\end{array}$ & $\begin{array}{l}\text { CCL3-5, CXCL10, CCL2, } \\
\text { CCL7, CCL1, CCL12, CXCL9 }\end{array}$ & {$[26]$} \\
\hline \multicolumn{5}{|l|}{ JEV } \\
\hline \multirow[t]{2}{*}{ Blood } & Human & Neutrophils & CXCL8, CCL5 & {$[79,80,82,83]$} \\
\hline & Mouse & Neutrophils & - & {$[81]$} \\
\hline CSF & Human & $\begin{array}{l}\text { Neutrophils/lymphocytes } \\
\quad(\text { CD4 > CD8), macrophages, B cells) }\end{array}$ & CCL5 and CXCL8 & {$[72,79]$} \\
\hline \multirow[t]{2}{*}{ Brain } & Human & Predominantly macrophages and $\mathrm{T}$ cells & - & [71] \\
\hline & Mouse & CD8 $+\mathrm{T}$ cells & CCL2-4, CXCL10-12, CCL12 & {$[74,75]$} \\
\hline \multicolumn{5}{|l|}{ TBEV } \\
\hline Blood & Human & - & CCL3, CXCL10, CXCL13 & {$[100,101]$} \\
\hline CSF & Human & $\mathrm{T}$ cells $(\mathrm{CD} 4>\mathrm{CD} 8)$, few $\mathrm{B}$ cells and NK cells & CCL2-3, CCL5, CXCL10-13 & {$[98,100,101,103,104]$} \\
\hline \multirow[t]{2}{*}{ Brain } & Human & Macrophages, $T$ cells $(\mathrm{CD} 8>\mathrm{CD} 4)$, few $\mathrm{B}$ cells & - & {$[95]$} \\
\hline & Mouse & Predominantly $\mathrm{CD} 8+\mathrm{T}$, some $\mathrm{CD} 4+\mathrm{T}$ & CCL2-CCL5, CXCL10 & {$[73,97]$} \\
\hline
\end{tabular}

precise importance of individual chemokines/receptors during WNV infection in vivo, we and others have primarily relied on the use of genetically deficient mouse models and, where possible, human epidemiological studies. Our current understanding of chemokine regulation and leukocyte trafficking during WNV infection is summarized in Fig. 1. Together, these data have started to reveal an integrated picture of how chemokines regulate specific cell populations in a time- and organ-specific manner.

\section{CCR5}

Among the most highly induced chemokines in the CNS following WNV infection are the CCR5 ligands, particularly CCL5 [24-26, 37]. Under steady-state conditions, CCR5 is expressed on many cell types, including activated $\mathrm{T}$ cells, a small percentage of monocytes, NK cells, and activated microglia. CCR5 is critically important during WNV infection, since CCR5-deficiency in WNV-infected mice resulted in uniform mortality by day 14 compared to wildtype mice, where the majority survived [26]. This dramatic increase in mortality was associated with an increase in CNS viral load and a decrease in all leukocyte subsets in the CNS (CD4+ and CD8 + T cells, monocytes, and NK cells) that are typically found in the CNS of WNV-infected wild-type mice (Fig. 1). Notably, clearance of virus from the spleen and numbers of IFN- $\gamma$ expressing splenocytes in the Ccr5deficient mice were identical to controls, suggesting that the defect was specific to the CNS. More precise analysis of the role of CCR5 in the periphery and the CNS is needed to fully understand the molecular role of this receptor in host defense.

Several epidemiologic studies have been conducted to evaluate the role of CCR5 in controlling WNV infection in humans as well [26, 27, 38-40]. These studies have analyzed the complete loss-of-function allele, CCR5432, among WNV-infected cohorts. In our initial studies, a strong association between CCR5 432 homozygosity (CCR5-deficient individuals) and symptomatic WNV disease was reported [26, 27, 40]. Compared to a reference cohort of uninfected random blood donors, where the frequency of CCR5 432 homozygosity was $\sim 1 \%$, the frequency among WNVinfected individuals with symptomatic disease (meningitis, encephalitis) was highly enriched ( $\sim-5 \%$; $p<0.0001)$. We also analyzed WNV-infected individuals identified through the American Red Cross blood supply, all of whom seroconverted and completed a questionnaire regarding symptom development in the 2 weeks following donation. Compared to false-positive control samples, CCR5 432 homozygotes were absent in the WNV seropositive individuals who remained completely asymptomatic. Among the WNV-infected individuals with symptoms, the frequency of CCR5 432 homozygosity was positively correlated with the number of symptoms reported following infection. Together, these data suggest that CCR5 is a strong host defense factor against WNV infection. In two recent follow-up studies, the 
results were less conclusive. A study conducted by Bigham et al. found a significant association with CCR5 32 and WNV infected individuals, which included both asymptomatic and symptomatic individuals [39]. When the symptomatic WNV-infected group was compared to asymptomatic or WNV-negative samples, no significant association was observed. This study may have been impacted by several factors: (1) $55 \%$ of the WNV-infected participants in their study were of self-reported European decent, while the control population was $99 \%$. Since the allele frequency of CCR5 432 is very low or absent among individuals of non-European decent, the analysis may have been biased [41]; (2) the "asymptomatic" WNV-infected participants in this study were classified based on the absence of symptoms in the week prior to donation. Since infected individuals develop symptoms in 2 weeks following viremia, some of these individuals likely developed symptoms; (3) unless the "asymptomatic" individuals were retested for seroconversion, this group is likely to contain a large percentage of false-positive samples since the WNV nucleic acid test used by blood banks has a high rate of false positivity [42, 43]. Another follow-up study by Loeb et al. has provided an analysis of WNV infection and CCR5 432 , and found no significant association. Although the data show a trend toward higher CCR5 32 homozygosity among WNVinfected individuals, the genotype frequency was much lower than previous reports. The lack of association observed in the latter studies may reflect differences in study design, choice of controls, or other factors regulated by CCR5. Future studies are greatly needed to determine the association of WNV and CCR5 432 .

Although the epidemiologic data suggest a critical function for CCR5 in controlling WNV pathogenesis, the current understanding of how this receptor functions in humans and mice remains poor. Since CCR5 is a leukocyte trafficking receptor, one possible mechanism by which CCR5 may confer resistance is by directing CCR5expressing leukocytes from the blood into the CNS. However, other CCR5-mediated neuroprotection mechanisms may also be involved and could include a role for CCR5 on activated microglia or for $\mathrm{T}$ cell activation in draining lymph nodes. Due to its role in HIV-1 entry, CCR5 is a logical and attractive therapeutic target (reviewed in [44]). As such, there are several CCR5 antagonists at various stages of development for the treatment of HIV infection and other inflammatory conditions [45]. Systemic blockade of CCR5 may sufficiently mimic genetic deficiency and promote susceptibility to WNV infection (Fig. 1). Maraviroc was FDA-approved in 2007, and two additional drugs are currently in clinical trials [46]. Chemokine analogues, such CCR5 antagonist PSC-RANTES, previously shown as an efficacious topical microbicide in the context of HIV, if used systemically, could increase pathogenesis of WNV (Fig. 1) [47]. The need to understand the precise role of CCR5 in host defense in this model is critical.

\section{CXCR3/CXCL10}

A key step in clearing WNV in the CNS is the recruitment of antigen-specific $\mathrm{CD} 8+\mathrm{T}$ lymphocytes into the brain parenchyma [48]. CD8-mediated clearance of WNV-infected neurons is perforin-dependent [49] and involves the induction of interferon-inducible Th1-specific chemokine CXCL10, which is expressed early within the CNS in mice [26, 49, 50]. Using genetically deficient mice and antibody neutralization approaches, Klein and colleagues showed that CXCL10 is critical for the recruitment of CXCR3-expressing $\mathrm{T}$ cells into the CNS and survival from WNV infection in mice [25]. Likewise, genetic deficiency of CXCR3 recapitulated this phenotype [50, 51]. Loss of either resulted in decreased T-cell recruitment to the CNS and increased viral burden and mortality. These data show the importance of CD8+ T-cell recruitment for this migratory step into the CNS and the requirement for effective clearance of WNV from infected neurons. In addition, the CXCR3 ligand CXCL9 is also induced in the CNS during WNV encephalitis, but the role of this chemokine has not yet been studied [25].

\section{CXCR4}

It is clear that the recruitment of $\mathrm{T}$ cells, both $\mathrm{CD} 4+$ and $\mathrm{CD} 8+$, into the CNS is an essential step for limiting WNV replication. However, studying the role of CXCR4, a chemokine receptor found expressed on $\mathrm{T}$ cells, NK cells, neutrophils, and several other leukocytes, along with its sole ligand CXCL12, in mice is difficult since genetic deficiency of this receptor or its ligand is lethal in utero [52]. Studies using the CXCR4-specific antagonist, AMD3100, have revealed an important migratory step of T cells into the WNV-infected CNS [29]. Expression analysis demonstrated CXCL12 on the microvasculature under uninfected conditions; this expression is downregulated during WNV encephalitis, suggesting a mechanism of CXCR4-mediated retention within the perivascular spaces of the CNS. In vivo administration of AMD3100, which blocks this interaction, allowed for the migration of $\mathrm{T}$ lymphocytes from perivascular space to the brain parenchyma and demonstrated enhanced viral clearance (Fig. 1). This reveals a possible role of CXCR4 in restricting T lymphocyte entry into the CNS to clear the virus, a mechanism similar to CXCR4's role in leukocyte retention in the bone marrow [53, 54]. Treatment of mice with AMD3100 was therapeutic, demonstrating the potential use 
for this drug in the treatment of WNV-infected individuals, a strategy that could function after the onset of symptoms. It may also be informative to test the changes in leukocyte mobilization stimulated by AMD3100. In mice and humans, AMD3100 is capable of mobilizing T cells (and other leukocytes), which may have also contributed to the resistance phenotype observed in these mice $[29,55,56]$.

\section{CCR2}

In addition to $\mathrm{T}$ cells, monocytes also comprise a high proportion of the leukocyte infiltrate during WNV encephalitis. It is generally accepted that blood monocytes are important cellular mediators of the innate immune response and can give rise to tissue macrophages and dendritic cells (DC). In mice and humans, high expression of CCR2 is associated with the "inflammatory" monocyte subset and correlates with their function of rapid recruitment into inflamed tissues. Monocytes may also play an important role during CNS injury since these cells have been demonstrated to migrate into the CNS to give rise to macrophages and microglia. However, their precise role in WNV infection may vary depending on the model. In particular, a recent study using a lethal intranasal model and a less pathogenic strain of WNV demonstrated that the majority of monocytes recruited to the CNS during encephalitis were the CCR2-expressing subset (>90\%), and these cells were capable of differentiating into both microglia and macrophages [57]. The authors found that delaying migration of these cells using anti-CCL2 neutralizing antibody prolonged survival, suggesting a CCR2dependent mechanism and a pathogenic role of monocytes recruited to the CNS. In contrast, WNV challenge by intraperitoneal inoculation has been reported to result in increased mortality when monocytes are depleted in vivo using clodronate-loaded liposomes, supporting a protective role for these cells [58].

Our studies in wild-type mice showed that WNV infection results in a fivefold induction of monocytes in the blood within the first 5 days postinfection (Table 1; Fig. 1). This was not observed for any other leukocyte subset and was CCR2-dependent since no monocytosis was observed in WNV-infected Ccr2-deficient mice [59]. Loss of CCR2 resulted in a marked increase in mortality and a large and selective reduction in Ly6c ${ }^{\text {hi }}$ monocyte accumulation in the brain. WNV infection in wild-type mice induced a strong and highly selective monocytosis in peripheral blood that was absent in $C c r 2^{-/-}$mice, which in contrast showed sustained monocytopenia. Adoptive transfer of an equal number of CCR2+ or CCR2- monocytes transferred by tail vein into WNV-infected $C c r 2^{-/-}$ recipient mice demonstrated CCR2 may be less critical for trafficking of monocytes from the blood to the brain. These data suggest that CCR2 mediates a highly selective peripheral blood monocytosis during WNV infection in mice that is critical for the accumulation of monocytes in the brain. The function of the CCR2 ligands, CCL2, CCL7, and CCL12, which are induced both in the CNS and blood in mice, remains unclear. Interestingly, serum analysis showed that induction of CCL2 and CCL7 in WNVinfected Ccr2-deficient mice was significantly increased, suggesting a role of these ligands in monocyte egress from the bone marrow. Future studies may include defining the functional role of monocytes during WNV encephalitis, immunophenotyping the cells that produce CCR2 ligands after viral entry into the CNS, and in vitro studies of monocyte/macrophage interaction with virally infected neurons. From a therapeutic standpoint, factors that stimulate monocytosis could be used for patients infected with WNV. These may include CCL2/7, granulocyte colony stimulated factor (G-CSF), and AMD3100, which have been shown to stimulate monocyte release from the bone marrow [55].

\section{CXCR2}

The role of neutrophils during WNV infection in humans and mice is currently unclear. In humans, neutrophil accumulation in the CSF is observed in patients with WNV encephalitis, and neutrophilic meningitis is seen in $\sim 50 \%$ of individuals presenting with WNV neuroinvasive disease [33, 60, 61]. In mice, neutrophils have been observed to accumulate in the CNS following WNV encephalitis, and a more recent study has evaluated neutrophil function in vivo using antibody depletion [59, 62]. Intriguingly, these data show that neutrophils can function to promote host defense or increase susceptibility, which depends on the timing of the neutrophil depletion. Neutrophil depletion using either Gr-1 or Ly6G antibodies 1 or 2 days following infection with WNV resulted in increased susceptibility, suggesting a critical role for controlling viral pathogenesis. Unexpectedly, if neutrophil depletion was administered 1 day prior to infection, the opposite results were observed: increased resistance. With regards to chemokine receptor usage during recruitment, neutrophils appear to function through CXCR2 and respond to ligands CXCL1 and CXCL2 produced in the skin (Fig. 1) [63]. Genetic deficiency for CXCR2 resulted in increased survival, supporting a pathogenic role of neutrophils early during infection. Future studies are needed to address the mechanism by which neutrophils mediate pathogenesis early in the infection and protection at later times, perhaps in the infected CNS. 


\section{Japanese encephalitis virus}

Japanese encephalitis was first recognized in humans and horses in 1871 and was the causative agent of a severe epidemic in 1924 in Japan. By the 1930s, the virus was found to transmit mainly by the Culex mosquitoes. Over the next 8 decades, the geographic area affected by JEV has been steadily increasing (reviewed in [3]). Birds have been implicated as being the main reservoir with pigs acting as amplifying hosts; humans and horses are accidental hosts. JEV constitutes the most significant cause of mosquitoborne encephalitis worldwide and is endemic throughout large regions of Central and Southeast Asia. In 2011, the World Health Organization (WHO) reported $\sim 67,900$ Japanese encephalitis (JE) cases with $\sim 75 \%$ of these occurring primarily in children between 0 and 14 years of age [64]. Similar to WNV, most infections with JEV are inapparent, with 1 symptomatic case for every $25-1,000$ infections $[65,66]$. The incubation period is approximately 5-15 days, and disease syndromes can range from nonspecific febrile illness, meningitis, or encephalitis. Among the symptomatic cases, fatality is high, estimated at $\sim 30 \%$, with more than half of the surviving individuals suffering from long-term severe neurological sequelae. Despite the availability of an effective vaccine, JEV continues to be an important health problem, especially in developing countries of South and Southeast Asia. In the absence of specific antiviral therapies, understanding the factors that govern susceptibility is a high priority [67-69].

After inoculation into the skin, JEV infection in vivo occurs similarly to WNV, with viremia following brief amplification within the dermal tissue and lymph nodes. Following entry into the CNS through an unknown mechanism, neurons are the primary targets of infection [70]. Postmortem samples show virus antigen localized to neurons primarily in the thalamus and brain stem, although lesions can also be found in the cortex, basal ganglia, cerebellum, and the anterior horn cells of the spinal cord. In humans, the brain parenchyma appears to be primarily infiltrated by macrophages, with few $\mathrm{T}$ cells; $\mathrm{T}$ and $\mathrm{B}$ cells appeared primarily in the perivascular space [71]. Cells in the CSF are predominantly neutrophils and $\mathrm{T}$ cells, although B cells and macrophages have also been detected [72]. Little is known with regards to the role of chemokines and JEV pathogenesis in humans or mice, and this is an area of great interest for future studies. CD8 $+\mathrm{T}$ cells are the predominant cell subset found in the brain parenchyma in mice; these cells may function pathogenically in this model. A recent study showed increased survival of JEVinfected CD8-deficient mice compared to wild-type mice [73, 74]. Microarray analysis on brain tissue of JEVinfected mice identified CCL2, CXCL10, and CCL4 to be the most highly induced chemokines [75]. This profile suggests the role of monocytes/macrophages and T cells, which is consistent with the predominance of $\mathrm{T}$ cells (and possibly macrophages/microglia) in the CNS parenchyma observed in mice and humans. CXCL10, which is often induced following viral infection, suggests a possible role for CXCR3 and T-cell migration into the CNS. Immunofluorescence assays on JEV-infected brain slices show an increase in CXCL10 expression in astrocytes that correlated with neuronal injury [76]. Thus, the increase in CXCL10 expression may be involved in regulating outcome of JEV-infected mice. Interestingly, the predominant CCR5 ligand induced in this model was CCL4. Smaller increases in expression were also observed for CCL3, CXCL1-2, and CXCL12. A second microarray study on JEV-infected mouse neuroblastoma cells demonstrated an increase in CXCL10, CCL5, and CXCL1 expression supporting the in vivo data [77].

\section{CXCL8}

Unlike WNV, where monocytosis is observed, JEV induces a neutrophil leukocytosis in mice infected intraperitoneally; this has also been observed in human infections as well [78-81]. Although not well studied in mice, significant induction of CXCL8 was observed in patient serum and CSF, which corresponded with the presence of neutrophils in the CSF [72, 79, 82]. Notably, significantly higher levels of CXCL8 were associated with patient mortality [82, 83]. Currently, no mouse studies have addressed the specific role of neutrophils in the context of $\mathrm{JEV}$; however, these cells may be quite pathogenic. Additional studies are required to fully understand the functional role of these cells in mice.

\section{CCL5}

Similar to CXCL8, an inverse correlation has been demonstrated for serum levels of CCL5 and survival among patients with JEV encephalitis [83]. This correlate for disease severity may contribute to pathogenesis, although the mechanism by which this occurs can only be speculated at this time. Since CCL5 binds to chemokine receptors found on activated $\mathrm{T}$ cells, and subsets of monocytes, dendritic cells, and NK cells, this suggests a potential immunopathogenic role for these cells during infection. Interestingly, immunophenotyping of the lymphocytes in the CSF shows a predominance of CD4+ T cells, with fewer CD8+ T, B cells, and macrophages [72]. In vitro work has demonstrated that microglia and astrocytes are the main cells that produce CCL5 during JEV infection [84]. In mice, microarray analysis shows an upregulation of CCL5 in the brain of JEV-infected mice [83, 85]. Given the critical role of CCR5 in WNV, a role for this receptor in 
host defense in a mouse model of JEV should be tested, along with in vivo neutralization of CCL5. Genotypephenotype analysis with loss-of-function mutation CCR5 432 in cohorts where this allele is prevalent will also be informative.

\section{Tick-borne encephalitis virus}

TBEV is the most notable member among the tick-borne flaviviruses and is a significant cause of yearly morbidity and mortality. Over the last few decades, tick-borne encephalitis (TBE) has become a growing public health concern in Europe and Asia with a marked increase in both the incidence and geographic distribution of TBEV, reflective of factors such as climate change, the expanding habitat of transmitting tick vectors, and global travel [86]. Despite the availability of an effective vaccine, a $400 \%$ increase in morbidity was observed in Europe in the last 30 years with confirmed cases of TBEV identified in several countries for the first time, suggesting a continued increase in TBE in the coming years [87-89]. Currently available is a formalininactivated whole virus vaccine that has been shown to be effective as seen in Austria where 90\% of the population has been vaccinated and yielded a significant decrease in the number of TBEV hospitalization [88].

Transmission of TBEV is typically through the bite of an infected Ixodes tick, although alternative modes of transmission, through ingestion of unpasteurized milk, can also occur [6]. The virus is amplified in rodent species, with humans serving as dead end hosts. Disease can range from mild meningitis to severe encephalitis and/or paralysis [86, 90]. Depending on the TBEV subtype, mortality rates can range from $12 \%$ (European strains) to 20-40 \% (Far Eastern strains). After deposition into the skin, initial replication of TBEV occurs in Langerhan's cells and neutrophils [9194]. The virus is then transported to the local draining lymph node, where primary amplification of the virus occurs. Following a brief viremia, virus can spread to various organs including the CNS where neurons are the primary targets [95]. During TBEV infection in mice, the presence of CD8+ $\mathrm{T}$ cells in the brain parenchyma is the primary pathologic feature, although the chemokines involved in this process are poorly characterized [95-97]. Our current understanding of TBEV-associated chemokine expression has been primarily through the analysis of human samples. During the early stage of TBE in humans, the majority of the cells recruited to the CNS is T lymphocytes and to a lesser extent B lymphocytes and NK cells [98]. Additionally, the presence of macrophages, CD8 + T cells, and CD4+ T cells has been observed in the brains of fatal human TBEV cases [95, 99]. Human TBE patients have elevated CCL3, CXCL10, and CXCL13 levels in the serum; CCL2, CCL3, CCL5, and
CXCL10-13 have been detected in CSF, perhaps implicating the role of a Th-1-mediated response and the trafficking of $\mathrm{T}$ lymphocytes into the CNS [100-102]. Moreover, CCL2 and CCL5 were found to be elevated in the CSF of TBE patients even after the acute symptoms subsided [103, 104]. Unlike JEV and WNV, TBEV induces CXCL13, a B-cell chemoattractant; studies evaluating the role of CXCR5 and B-cell trafficking and maturation may be insightful. In a recent study of TBEV infection in mice, several chemokines have been shown to be strongly upregulated in the brain, with high induction of CXCL10, CXCL11, CCL5, CCL2, CCL3, and CCL4 [96]. This is followed by the appearance of peripheral leukocytes, of which CD8+ T cells appear in the highest numbers. Whether this model faithfully recapitulates human infections is not clear; many more studies evaluating the exact function of specific chemokine receptors are greatly needed.

\section{CCR5}

Given the similarities in the pathogenesis of WNV and TBEV, Kindberg et al. [105] evaluated the role of CCR5 in the context of TBEV infection in humans. Their study evaluated a cohort of Lithuanian patients infected with TBEV and demonstrated an increase in CCR5 432 allele frequency as well as homozygous frequency among TBEV patients with encephalitis compared with TBEV-negative meningoencephalitis cases or healthy age-, ethnicity- and geographically matched controls. Although their sample size was small, the CCR5 432 allele frequency was positively correlated with severity of disease. Although only a single study, it suggests that loss of CCR5 may predispose individuals to severe outcome from TBEV infection and warrants further investigation both in the mouse model and in larger cohorts.

\section{Conclusions}

Neurotropic flaviviruses represent a major unmet medical challenge since there are no specific and effective therapies available for WNV, JEV, TBEV, or any other member of the Flavivirus genus. Changes in climate, economy, politics, and globalization are among the factors that cause renewed concern due to the uncertainty of how these epidemics will progress in the coming years. Neuronal delivery limitations and the BBB often compromise the treatment of these diseases, and since even the most promising antivirals for WNV appear to be ineffective once symptoms develop (reviewed in [106]), alternatives based on precise understanding of the host response are a high priority. 
Leukocyte trafficking events are integrally involved in the complex dynamics between host and pathogen. For WNV, a great deal of research has been conducted to evaluate not only cell migration events during the course of infection, but the specific chemokines and receptors involved. Trafficking of cells from the blood to the CNS is a key step critical for survival, although many infectiontriggered trafficking events are equally important, starting with the migration of dendritic cells to the site of infection to the draining lymph nodes, neutrophil and monocyte mobilization from the bone marrow, and their dispatchment into the CNS. Based on this precise understanding, targets for drug intervention can now be envisioned. A great deal less is known about JEV and TBEV; however, JEV appears to trigger a specific neutrophilia, and increased CCL5 and CXCL8 correlate with poor prognosis. Future studies evaluating the role of neutrophils during JEV infection, and the possible role of B-cell chemokines in TBEV infection are interesting future directions. Due to biosafety restrictions, few studies have analyzed the role of chemokines and TBEV; perhaps, addressing questions using Langat virus (LGTV), a closely related tick-borne flavivirus, may provide some answers in the interim [107]. More studies are needed to characterize the specific cell subsets in the CNS, their function in pathogenesis, and their role in viral clearance.

Although there is great redundancy in the chemokine system, it appears that clearance of WNV requires a function of CCR5 that cannot be compensated for by a related receptor. In fact, there is evidence that increased susceptibility due to CCR5 deficiency may extend to related flaviviruses. Evidence for this comes from a recent study that showed that CCR5 deficiency is also associated with TBEV as well as the inverse correlation in CCL5 levels in serum and CSF with survival from JEV. Given the similarities with WNV, a role of CCR5 in a mouse model of JEV and TBEV would support the hypothesis that CCR5 may function globally in controlling neuroinvasive disease caused by flaviviruses. Future studies in mice should evaluate this in the context of JEV and TBEV as well as in humans. Based on the epidemiologic studies on CCR5 432 and WNV infection, more studies are needed to determine whether homozygosity for this allele is involved in susceptibility; rigorous attention should be placed on defining the WNV-infected individuals as well as control populations used, and interpretations of these studies should be cautious. This is particularly important given that CCR5 deficiency (CCR5 $\triangle 32$ homozygotes) is found in 1-2 \% of Caucasian individuals worldwide and the introduction of Maraviroc (CCR5 antagonists) for the treatment of HIV and other diseases [108]. Chronic use of such blocking agents is likely to promote the pathogenesis of WNV, TBEV, and possibly other pathogens.
Acknowledgments The authors would like to thank Philip M. Murphy for critical reading of the manuscript. Supported by the National Institutes of Health Grant K22A1087816-01.

\section{References}

1. Hayes EB, Gubler DJ. West Nile virus: epidemiology and clinical features of an emerging epidemic in the United States. Annu Rev Med. 2006;57:181-94. doi:10.1146/annurev.med.57. 121304.131418.

2. Lanciotti RS, Roehrig JT, Deubel V, Smith J, Parker M, Steele $\mathrm{K}$, et al. Origin of the West Nile virus responsible for an outbreak of encephalitis in the northeastern United States. Science. 1999;286(5448):2333-7.

3. Misra UK, Kalita J. Overview: Japanese encephalitis. Prog Neurobiol. 2010;91(2):108-20. doi:10.1016/j.pneurobio.2010. 01.008 .

4. Charrel RN, Attoui H, Butenko AM, Clegg JC, Deubel V, Frolova TV, et al. Tick-borne virus diseases of human interest in Europe. Clin Microbiol Infect. 2004;10(12):1040-55. doi: 10.1111/j.1469-0691.2004.01022.x.

5. Iwamoto M, Jernigan DB, Guasch A, Trepka MJ, Blackmore CG, Hellinger WC, et al. Transmission of West Nile virus from an organ donor to four transplant recipients. N Engl J Med. 2003;348(22):2196-203. doi:10.1056/NEJMoa022987.

6. Balogh Z, Egyed L, Ferenczi E, Ban E, Szomor KN, Takacs M, et al. Experimental infection of goats with tick-borne encephalitis virus and the possibilities to prevent virus transmission by raw goat milk. Intervirology. 2011. doi:10.1159/000324023.

7. Bakhvalova VN, Potapova OF, Panov VV, Morozova OV. Vertical transmission of tick-borne encephalitis virus between generations of adapted reservoir small rodents. Virus Res. 2009;140(1-2):172-8. doi:10.1016/j.virusres.2008.12.001.

8. Smithburn KC, Huges TP, Burke AW, Paul JH. A neurotropic virus isolated from the blood of a native Ugandna. Am J Trop Med Hyg. 1940;20:471-92.

9. Weaver SC, Barrett AD. Transmission cycles, host range, evolution and emergence of arboviral disease. Nat Rev Microbiol. 2004;2(10):789-801. doi:10.1038/nrmicro1006.

10. Debiasi RL. West nile virus neuroinvasive disease. Curr Infect Dis Rep. 2011;13(4):350-9. doi:10.1007/s11908-011-0193-9.

11. Nash D, Mostashari F, Fine A, Miller J, O’Leary D, Murray K, et al. The outbreak of West Nile virus infection in the New York City area in 1999. N Engl J Med. 2001;344(24):1807-14. doi: 10.1056/nejm200106143442401.

12. Hayes EB, Komar N, Nasci RS, Montgomery SP, O'Leary DR, Campbell GL. Epidemiology and transmission dynamics of West Nile virus disease. Emerg Infect Dis. 2005;11(8):1167-73.

13. CDC. West Nile Virus Statistics, Surveillance, and Control Archive. http://www.cdc.gov/ncidod/dvbid/westnile/surv\&control. htm. 2011.

14. Sejvar JJ. The long-term outcomes of human West Nile virus infection. Clin Infect Dis. 2007;44(12):1617-24. doi:10.1086/ 518281.

15. Brien JD, Uhrlaub JL, Hirsch A, Wiley CA, Nikolich-Zugich J. Key role of $T$ cell defects in age-related vulnerability to West Nile virus. J Exp Med. 2009;206(12):2735-45. doi:10.1084/ jem.20090222.

16. Byrne SN, Halliday GM, Johnston LJ, King NJ. Interleukin-1beta but not tumor necrosis factor is involved in West Nile virusinduced Langerhans cell migration from the skin in C57BL/6 mice. J Invest Dermatol. 2001;117(3):702-9. doi:10.1046/ j.0022-202x.2001.01454.x.

17. Garcia-Tapia D, Hassett DE, Mitchell WJ Jr, Johnson GC, Kleiboeker SB. West Nile virus encephalitis: sequential 
histopathological and immunological events in a murine model of infection. J Neurovirol. 2007;13(2):130-8. doi:10.1080/ 13550280601187185.

18. Johnston LJ, Halliday GM, King NJ. Langerhans cells migrate to local lymph nodes following cutaneous infection with an arbovirus. J Invest Dermatol. 2000;114(3):560-8. doi:10.1046/ j.1523-1747.2000.00904.x.

19. Wang T, Town T, Alexopoulou L, Anderson JF, Fikrig E, Flavell RA. Toll-like receptor 3 mediates West Nile virus entry into the brain causing lethal encephalitis. Nat Med. 2004;10(12): 1366-73. doi:10.1038/nm1140.

20. Xiao SY, Guzman H, Zhang H, Travassos da Rosa AP, Tesh RB. West Nile virus infection in the golden hamster (Mesocricetus auratus): a model for West Nile encephalitis. Emerg Infect Dis. 2001;7(4):714-21.

21. Guarner J, Shieh WJ, Hunter S, Paddock CD, Morken T, Campbell GL, et al. Clinicopathologic study and laboratory diagnosis of 23 cases with West Nile virus encephalomyelitis. Hum Pathol. 2004;35(8):983-90.

22. Shieh WJ, Guarner J, Layton M, Fine A, Miller J, Nash D, et al. The role of pathology in an investigation of an outbreak of West Nile encephalitis in New York, 1999. Emerg Infect Dis. 2000; 6(4):370-2.

23. Shrestha B, Gottlieb D, Diamond MS. Infection and injury of neurons by West Nile encephalitis virus. J Virol. 2003;77(24): 13203-13.

24. Shirato K, Kimura T, Mizutani T, Kariwa H, Takashima I. Different chemokine expression in lethal and non-lethal murine West Nile virus infection. J Med Virol. 2004;74(3):507-13. doi: 10.1002/jmv.20205.

25. Klein RS, Lin E, Zhang B, Luster AD, Tollett J, Samuel MA, et al. Neuronal CXCL10 directs CD8+ T-cell recruitment and control of West Nile virus encephalitis. J Virol. 2005;79(17): 11457-66. doi:10.1128/jvi.79.17.11457-11466.2005.

26. Glass WG, Lim JK, Cholera R, Pletnev AG, Gao JL, Murphy PM. Chemokine receptor CCR5 promotes leukocyte trafficking to the brain and survival in West Nile virus infection. J Exp Med. 2005;202(8):1087-98. doi:10.1084/jem.20042530.

27. Lim JK, McDermott DH, Lisco A, Foster GA, Krysztof D, Follmann D, et al. CCR5 deficiency is a risk factor for early clinical manifestations of West Nile virus infection but not for viral transmission. J Infect Dis. 2010;201(2):178-85. doi:10.1086/649426.

28. Tobler LH, Cameron MJ, Lanteri MC, Prince HE, Danesh A, Persad D, et al. Interferon and interferon-induced chemokine expression is associated with control of acute viremia in West Nile virus-infected blood donors. J Infect Dis. 2008;198(7): 979-83. doi:10.1086/591466.

29. McCandless EE, Zhang B, Diamond MS, Klein RS. CXCR4 antagonism increases $\mathrm{T}$ cell trafficking in the central nervous system and improves survival from West Nile virus encephalitis. Proc Natl Acad Sci USA. 2008;105(32):11270-5. doi:10.1073/ pnas.0800898105.

30. Cheeran MC, Hu S, Sheng WS, Rashid A, Peterson PK, Lokensgard JR. Differential responses of human brain cells to West Nile virus infection. J Neurovirol. 2005;11(6):512-24. doi:10. 1080/13550280500384982.

31. Burton JM, Kern RZ, Halliday W, Mikulis D, Brunton J, Fearon $\mathrm{M}$, et al. Neurological manifestations of West Nile virus infection. Can J Neurol Sci. 2004;31(2):185-93.

32. Sampson BA, Armbrustmacher V. West Nile encephalitis: the neuropathology of four fatalities. Ann NY Acad Sci. 2001;951: 172-8.

33. Tyler KL, Pape J, Goody RJ, Corkill M, Kleinschmidt-DeMasters BK. CSF findings in 250 patients with serologically confirmed West Nile virus meningitis and encephalitis.
Neurology. 2006;66(3):361-5. doi:10.1212/01.wnl.0000195890. 70898.1f.

34. Jeha LE, Sila CA, Lederman RJ, Prayson RA, Isada CM, Gordon SM. West Nile virus infection: a new acute paralytic illness. Neurology. 2003;61(1):55-9.

35. Procop GW, Yen-Lieberman B, Prayson RA, Gordon SM. Mollaret-like cells in patients with West Nile virus infection. Emerg Infect Dis. 2004;10(4):753-4.

36. Shrestha B, Diamond MS. Role of CD8+ T cells in control of West Nile virus infection. J Virol. 2004;78(15):8312-21. doi: 10.1128/jvi.78.15.8312-8321.2004.

37. Glass WG, McDermott DH, Lim JK, Lekhong S, Yu SF, Frank WA, et al. CCR5 deficiency increases risk of symptomatic West Nile virus infection. J Exp Med. 2006;203(1):35-40. doi: 10.1084/jem.20051970.

38. Loeb M, Eskandarian S, Rupp M, Fishman N, Gasink L, Patterson J, et al. Genetic variants and susceptibility to neurological complications following West Nile virus infection. J Infect Dis. 2011;204(7):1031-7. doi:10.1093/infdis/jir493.

39. Bigham AW, Buckingham KJ, Husain S, Emond MJ, Bofferding $\mathrm{KM}$, Gildersleeve $\mathrm{H}$, et al. Host genetic risk factors for West Nile virus infection and disease progression. PLoS ONE. 2011;6(9):e24745. doi:10.1371/journal.pone.0024745.

40. Lim JK, Louie CY, Glaser C, Jean C, Johnson B, Johnson H, et al. Genetic deficiency of chemokine receptor CCR5 is a strong risk factor for symptomatic West Nile virus infection: a metaanalysis of 4 cohorts in the US epidemic. J Infect Dis. 2008;197(2):262-5. doi:10.1086/524691.

41. Martinson JJ, Chapman NH, Rees DC, Liu YT, Clegg JB. Global distribution of the CCR5 gene 32-basepair deletion. Nat Genet. 1997;16(1):100-3. doi:10.1038/ng0597-100.

42. Orton SL, Stramer SL, Dodd RY. Self-reported symptoms associated with West Nile virus infection in RNA-positive blood donors. Transfusion. 2006;46(2):272-7. doi:10.1111/j.1537-29 95.2006.00710.x.

43. Stramer SL, Fang CT, Foster GA, Wagner AG, Brodsky JP, Dodd RY. West Nile virus among blood donors in the United States, 2003 and 2004. N Engl J Med. 2005;353(5):451-9. doi: 10.1056/NEJMoa044333.

44. Lim JK, Glass WG, McDermott DH, Murphy PM. CCR5: no longer a "good for nothing" gene-chemokine control of West Nile virus infection. Trends Immunol. 2006;27(7):308-12. doi: 10.1016/j.it.2006.05.007.

45. Gilliam BL, Riedel DJ, Redfield RR. Clinical use of CCR5 inhibitors in HIV and beyond. J Transl Med. 2011;9(Suppl 1):S9. doi:10.1186/1479-5876-9-s1-s9.

46. Liu T, Weng Z, Dong X, Hu Y. Recent advances in the development of small-molecule CCR5 inhibitors for HIV. Mini Rev Med Chem. 2010;10(13):1277-92.

47. Lederman MM, Veazey RS, Offord R, Mosier DE, Dufour J, Mefford M, et al. Prevention of vaginal SHIV transmission in rhesus macaques through inhibition of CCR5. Science. 2004;306(5695):485-7. doi:10.1126/science.1099288.

48. McGavern DB, Homann D, Oldstone MB. T cells in the central nervous system: the delicate balance between viral clearance and disease. J Infect Dis. 2002;186(Suppl 2):S145-51. doi:10. $1086 / 344264$.

49. Shrestha B, Samuel MA, Diamond MS. CD8+ T cells require perforin to clear West Nile virus from infected neurons. J Virol. 2006;80(1):119-29. doi:10.1128/jvi.80.1.119-129.2006.

50. Zhang B, Patel J, Croyle M, Diamond MS, Klein RS. TNFalpha-dependent regulation of CXCR3 expression modulates neuronal survival during West Nile virus encephalitis. J Neuroimmunol. 2010;224(1-2):28-38. doi:10.1016/j.jneuroim.2010. 05.003 . 
51. Zhang B, Chan YK, Lu B, Diamond MS, Klein RS. CXCR3 mediates region-specific antiviral $\mathrm{T}$ cell trafficking within the central nervous system during West Nile virus encephalitis. J Immunol. 2008;180(4):2641-9.

52. Tachibana $\mathrm{K}$, Hirota $\mathrm{S}$, Iizasa $\mathrm{H}$, Yoshida $\mathrm{H}$, Kawabata $\mathrm{K}$, Kataoka Y, et al. The chemokine receptor CXCR4 is essential for vascularization of the gastrointestinal tract. Nature. 1998; 393(6685):591-4. doi:10.1038/31261.

53. Link DC. Neutrophil homeostasis: a new role for stromal cellderived factor-1. Immunol Res. 2005;32(1-3):169-78. doi: 10.1385/ir:32:1-3:169.

54. Eash KJ, Means JM, White DW, Link DC. CXCR4 is a key regulator of neutrophil release from the bone marrow under basal and stress granulopoiesis conditions. Blood. 2009;113(19): 4711-9. doi:10.1182/blood-2008-09-177287.

55. Capoccia BJ, Shepherd RM, Link DC. G-CSF and AMD3100 mobilize monocytes into the blood that stimulate angiogenesis in vivo through a paracrine mechanism. Blood. 2006;108(7): 2438-45. doi:10.1182/blood-2006-04-013755.

56. McDermott DH, Liu Q, Ulrick J, Kwatemaa N, Anaya-O’Brien S, Penzak SR, et al. The CXCR4 antagonist plerixafor corrects panleukopenia in patients with WHIM syndrome. Blood. 2011;118(18):4957-62. doi:10.1182/blood-2011-07-368084.

57. Getts DR, Terry RL, Getts MT, Muller M, Rana S, Shrestha B, et al. Ly6c+ "inflammatory monocytes" are microglial precursors recruited in a pathogenic manner in West Nile virus encephalitis. J Exp Med. 2008;205(10):2319-37. doi:10.1084/ jem.20080421.

58. Ben-Nathan D, Huitinga I, Lustig S, van Rooijen N, Kobiler D. West Nile virus neuroinvasion and encephalitis induced by macrophage depletion in mice. Arch Virol. 1996;141(3-4):459-69.

59. Lim JK, Obara CJ, Rivollier A, Pletnev AG, Kelsall BL, Murphy PM. Chemokine receptor Ccr2 is critical for monocyte accumulation and survival in West Nile virus encephalitis. J Immunol. 2011;186(1):471-8. doi:10.4049/jimmunol.1003003.

60. Rawal A, Gavin PJ, Sturgis CD. Cerebrospinal fluid cytology in seasonal epidemic West Nile virus meningo-encephalitis. Diagn Cytopathol. 2006;34(2):127-9. doi:10.1002/dc.20410.

61. Brehin AC, Mouries J, Frenkiel MP, Dadaglio G, Despres P, Lafon $\mathrm{M}$, et al. Dynamics of immune cell recruitment during West Nile encephalitis and identification of a new CD19+ B220-BST-2+ leukocyte population. J Immunol. 2008;180(10): 6760-7.

62. Bai F, Kong KF, Dai J, Qian F, Zhang L, Brown CR, et al. A paradoxical role for neutrophils in the pathogenesis of West Nile virus. J Infect Dis. 2010;202(12):1804-12. doi:10.1086/657416.

63. Kish DD, Li X, Fairchild RL. CD8 T cells producing IL-17 and IFN-gamma initiate the innate immune response required for responses to antigen skin challenge. J Immunol. 2009;182(10): 5949-59. doi:10.4049/jimmunol.0802830.

64. Campbell GL, Hills SL, Fischer M, Jacobson JA, Hoke CH, Hombach JM, et al. Estimated global incidence of Japanese encephalitis: a systemic review. Bull World Health Organ. 2011;89(10):766-74A-74E. doi:10.2471/blt.10.085233.

65. Halstead SB, Grosz CR. Subclinical Japanese encephalitis. I. Infection of Americans with limited residence in Korea. Am J Hyg. 1962;75:190-201.

66. Huang CH. Studies of Japanese encephalitis in China. Adv Virus Res. 1982;27:71-101.

67. Hammon WM, Sather GE, Mc CH. Serologic survey of Japanese $\mathrm{B}$ encephalitis virus infection in birds in Japan. Am J Hyg. 1958;67(1):118-33.

68. Cross JH, Lien JC, Huang WC, Lien SC, Chiu SF. Japanese encephalitis virus surveillance in Taiwan. II. Isolations from mosquitoes and bats in Taipei area 1969-1970. Taiwan Yi Xue Hui Za Zhi. 1971;70(12):681-6.
69. Rosen L. The natural history of Japanese encephalitis virus. Annu Rev Microbiol. 1986;40:395-414. doi:10.1146/annurev. mi.40.100186.002143.

70. Yasui K. Neuropathogenesis of Japanese encephalitis virus. J Neurovirol. 2002;8(Suppl 2):112-4. doi:10.1080/13550280290 168019.

71. Johnson RT, Burke DS, Elwell M, Leake CJ, Nisalak A, Hoke $\mathrm{CH}$, et al. Japanese encephalitis: immunocytochemical studies of viral antigen and inflammatory cells in fatal cases. Ann Neurol. 1985;18(5):567-73. doi:10.1002/ana.410180510.

72. Johnson RT, Intralawan P, Puapanwatton S. Japanese encephalitis: identification of inflammatory cells in cerebrospinal fluid. Ann Neurol. 1986;20(6):691-5. doi:10.1002/ana.410200607.

73. Ruzek D, Salat J, Palus M, Gritsun TS, Gould EA, Dykova I, et al. CD8+ T-cells mediate immunopathology in tick-borne encephalitis. Virology. 2009;384(1):1-6. doi:10.1016/j.virol. 2008.11.023.

74. Fujii Y, Kitaura K, Nakamichi K, Takasaki T, Suzuki R, Kurane I. Accumulation of T-cells with selected T-cell receptors in the brains of Japanese encephalitis virus-infected mice. Jpn J Infect Dis. 2008;61(1):40-8.

75. Yang Y, Ye J, Yang X, Jiang R, Chen H, Cao S. Japanese encephalitis virus infection induces changes of mRNA profile of mouse spleen and brain. Virol J. 2011;8:80. doi:10.1186/1743$422 \mathrm{x}-8-80$.

76. Bhowmick S, Duseja R, Das S, Appaiahgiri MB, Vrati S, Basu A. Induction of IP-10 (CXCL10) in astrocytes following Japanese encephalitis. Neurosci Lett. 2007;414(1):45-50. doi: 10.1016/j.neulet.2006.11.070.

77. Gupta N, Santhosh SR, Babu JP, Parida MM, Rao PV. Chemokine profiling of Japanese encephalitis virus-infected mouse neuroblastoma cells by microarray and real-time RT-PCR: implication in neuropathogenesis. Virus Res. 2010;147(1):10712. doi:10.1016/j.virusres.2009.10.018.

78. Mathur A, Bharadwaj M, Kulshreshtha R, Rawat S, Jain A, Chaturvedi UC. Immunopathological study of spleen during Japanese encephalitis virus infection in mice. Br J Exp Pathol. 1988;69(3):423-32.

79. Chung CC, Lee SS, Chen YS, Tsai HC, Wann SR, Kao CH, et al. Acute flaccid paralysis as an unusual presenting symptom of Japanese encephalitis: a case report and review of the literature. Infection. 2007;35(1):30-2. doi:10.1007/s15010-0076038-7.

80. Chaturvedi UC, Mathur A, Tandon P, Natu SM, Rajvanshi S, Tandon HO. Variable effect on peripheral blood leucocytes during JE virus infection of man. Clin Exp Immunol. 1979;38(3):492-8.

81. Mathur A, Khanna N, Chaturvedi UC. Breakdown of bloodbrain barrier by virus-induced cytokine during Japanese encephalitis virus infection. Int J Exp Pathol. 1992;73(5):60311.

82. Singh A, Kulshreshtha R, Mathur A. Secretion of the chemokine interleukin-8 during Japanese encephalitis virus infection. J Med Microbiol. 2000;49(7):607-12.

83. Winter PM, Dung NM, Loan HT, Kneen R, Wills B, le Thu T, et al. Proinflammatory cytokines and chemokines in humans with Japanese encephalitis. J Infect Dis. 2004;190(9):1618-26. doi: $10.1086 / 423328$.

84. Chen CJ, Chen JH, Chen SY, Liao SL, Raung SL. Upregulation of RANTES gene expression in neuroglia by Japanese encephalitis virus infection. J Virol. 2004;78(22):12107-19. doi: 10.1128/jvi.78.22.12107-12119.2004.

85. Gupta N, Rao PV. Transcriptomic profile of host response in Japanese encephalitis virus infection. Virol J. 2011;8:92. doi: 10.1186/1743-422x-8-92.

86. Gritsun TS, Lashkevich VA, Gould EA. Tick-borne encephalitis. Antivir Res. 2003;57(1-2):129-46. 
87. Csango PA, Blakstad E, Kirtz GC, Pedersen JE, Czettel B. Tickborne encephalitis in southern Norway. Emerg Infect Dis. 2004; 10(3):533-4.

88. Mansfield KL, Johnson N, Phipps LP, Stephenson JR, Fooks AR, Solomon T. Tick-borne encephalitis virus-a review of an emerging zoonosis. J Gen Virol. 2009;90(Pt 8):1781-94. doi: 10.1099/vir.0.011437-0.

89. Suss J. Tick-borne encephalitis in Europe and beyond-the epidemiological situation as of 2007. Euro Surveill. 2008;13(26):1-8.

90. Gritsun TS, Nuttall PA, Gould EA. Tick-borne flaviviruses. Adv Virus Res. 2003;61:317-71.

91. Albrecht P. Pathogenesis of neurotropic arbovirus infections. Curr Top Microbiol Immunol. 1968;43:44-91.

92. Haglund M, Gunther G. Tick-borne encephalitis-pathogenesis, clinical course and long-term follow-up. Vaccine. 2003; 21(Suppl 1):S11-8.

93. Labuda M, Austyn JM, Zuffova E, Kozuch O, Fuchsberger N, Lysy J, et al. Importance of localized skin infection in tick-borne encephalitis virus transmission. Virology. 1996;219(2):357-66. doi:10.1006/viro.1996.0261.

94. Wu ML, Warren DJ, Jones VA. Ticked off: ixodes. Arch Pathol Lab Med. 2000;124(6):925. doi:10.1043/0003-9985(2000)124 $<0925$ :toi $>2.0$. co; 2 .

95. Gelpi E, Preusser M, Garzuly F, Holzmann H, Heinz FX, Budka H. Visualization of Central European tick-borne encephalitis infection in fatal human cases. J Neuropathol Exp Neurol. 2005;64(6):506-12.

96. Ruzek D, Salat J, Singh SK, Kopecky J. Breakdown of the blood-brain barrier during tick-borne encephalitis in mice is not dependent on CD8+ T-cells. PLoS ONE. 2011;6(5):e20472. doi:10.1371/journal.pone.0020472.

97. Hayasaka D, Nagata N, Fujii Y, Hasegawa H, Sata T, Suzuki R, et al. Mortality following peripheral infection with tick-borne encephalitis virus results from a combination of central nervous system pathology, systemic inflammatory and stress responses. Virology. 2009;390(1):139-50. doi:10.1016/j.virol.2009.04.026.

98. Holub M, Kluckova Z, Beran O, Aster V, Lobovska A. Lymphocyte subset numbers in cerebrospinal fluid: comparison of tick-borne encephalitis and neuroborreliosis. Acta Neurol Scand. 2002;106(5):302-8.

99. Gelpi E, Preusser M, Laggner U, Garzuly F, Holzmann H, Heinz FX, et al. Inflammatory response in human tick-borne encephalitis: analysis of postmortem brain tissue. J Neurovirol. 2006;12(4): 322-7. doi:10.1080/13550280600848746.

100. Zajkowska J, Moniuszko-Malinowska A, Pancewicz SA, Muszynska-Mazur A, Kondrusik M, Grygorczuk S, et al. Evaluation of CXCL10, CXCL11, CXCL12 and CXCL13 chemokines in serum and cerebrospinal fluid in patients with tick borne encephalitis (TBE). Adv Med Sci. 2011;56:1-7. doi:10.2478/v10039-0110033-z.

101. Grygorczuk S, Zajkowska J, Swierzbinska R, Pancewicz S, Kondrusik M, Hermanowska-Szpakowicz T. Elevated concentration of the chemokine CCL3 (MIP-1alpha) in cerebrospinal fluid and serum of patients with tick borne encephalitis. Adv Med Sci. 2006;51:340-4.

102. Lepej SZ, Misic-Majerus L, Jeren T, Rode OD, Remenar A, Sporec V, et al. Chemokines CXCL10 and CXCL11 in the cerebrospinal fluid of patients with tick-borne encephalitis. Acta Neurol Scand. 2007;115(2):109-14. doi:10.1111/j.1600-0404. 2006.00726.x.

103. Michalowska-Wender G, Losy J, Kondrusik M, Zajkowska J, Pancewicz S, Grygorczuk S, et al. Evaluation of soluble platelet cell adhesion molecule sPECAM-1 and chemokine MCP-1 (CCL2) concentration in CSF of patients with tick-borne encephalitis]. Pol Merkur Lekarski. 2006;20(115):46-8.

104. Grygorczuk S, Zajkowska J, Swierzbinska R, Pancewicz S, Kondrusik M, Hermanowska-Szpakowicz T. Concentration of the beta-chemokine CCL5 (RANTES) in cerebrospinal fluid in patients with tick-borne encephalitis]. Neurol Neurochir Pol. 2006;40(2):106-11.

105. Kindberg E, Mickiene A, Ax C, Akerlind B, Vene S, Lindquist $\mathrm{L}$, et al. A deletion in the chemokine receptor 5 (CCR5) gene is associated with tickborne encephalitis. J Infect Dis. 2008; 197(2):266-9. doi:10.1086/524709.

106. Diamond MS. Progress on the development of therapeutics against West Nile virus. Antiviral Res. 2009;83(3):214-27. doi: 10.1016/j.antiviral.2009.05.006.

107. Smith CE. A virus resembling Russian spring-summer encephalitis virus from an ixodid tick in Malaya. Nature. 1956; 178(4533):581-2.

108. Gulick RM, Lalezari J, Goodrich J, Clumeck N, DeJesus E, Horban A, et al. Maraviroc for previously treated patients with R5 HIV-1 infection. N Engl J Med. 2008;359(14):1429-41. doi: 10.1056/NEJMoa0803152. 